\title{
Alternating increased unilateral vocal cord uptake in a patient with metastatic breast cancer
}

\author{
Volkan Beylergil $^{1 *}$ and Jorge A Carrasquillo ${ }^{2}$ \\ ${ }^{1}$ The Metrohealth Campus of Case Western Reserve University, Department of Radiology, 2500 Metrohealth Drive Cleveland, OH 44108, USA \\ ${ }^{2}$ Department of Radiology, Molecular Imaging and Therapy Service, 1275 York Avenue New York, NY 10065, USA
}

\begin{abstract}
We present a 58 year old female with stage IV breast cancer, status bilateral mastectomies that underwent FDG PET/CT for evaluation of extent of disease. The PET scan shows increased uptake in the left vocal cord (SUV max 3.9), consistent with right vocal cord paralysis presumably from a hypermetabolic right paratracheal node involving the recurrent laryngeal. Clinically hoarseness was confirmed and intermittent aspiration of liquids led to treatment with injection of CAHA in the right vocal cord.
\end{abstract}

A follow up restaging scan performed a year after the initial scan showed FDG uptake in the injected vocal cord overlying the area of dense material in the injected side (SUVmax 9.2) and resolution of previously noted hypermetabolic metastasis in the paratracheal region and bone metastasis(data not shown).

\section{Introduction}

Vocal cord paralysis(VCP) is a fairly common and usually a harbinger of an underlying significant illness. Unilateral increased FDG uptake in the unaffected cord is a common pitfall and have sometimes been misinterpreted as primary or secondary malignancy especially in the pre-PET/CT era. Additionally, local injection therapies for VCP can cause pitfalls and complicate the interpretation.

\section{Case report}

We present a 58 year old female with stage IV breast cancer, status bilateral mastectomies who underwent FDG PET/CT for follow up. The PET scan showed increased uptake in the left vocal cord (SUV max 3.9), consistent with right vocal cord paralysis presumably from a hypermetabolic right paratracheal node involving the recurrent laryngeal nerve (Figure 1A). Post-treatment axial PET, CT and fused PET/CT images showed focal intense FDG uptake in the right vocal cord at the CAHA injection site(SUV 9.2) (Figure 1B). Baseline FDG $\mathrm{PET} / \mathrm{CT}$ scan showed a hypermetabolic right paratracheal adenopathy, the possible culprit for recurrent laryngeal nerve palsy.

\section{Literature review and discussion}

In a retrospective study, Chen et al have reported neoplastic disease as the second most common cause of VCP [1], with the traumatic causes being the most common cause. The unilateral increased FDG uptake in the unaffected, non-paralyzed vocal cord has been well demonstrated [2] and attributed to compensatory increased workload of the normal vocal cord. To improve symptoms, medialization of the vocal cord is often performed using a variety of materials including teflon, fat, collagen, hyaluronic acid, calcium hydroxyapatite gel(CAHA), and polydimethylsiloxane gel. Some of these injections have been associated with increased FDG uptake in the ipsilateral injected vocal cord $[3,4]$ (Figure 2).

Blodgett et al. [5] have summarized common physiologic variants and pitfalls of combined PET/CT in the head and neck in an excellent review. It is important to distinguish unilateral vocal cord uptake from vocal cord neoplasms. This patient illustrates both the physiologic contralateral normal vocal cord uptake during vocal cord paralysis as well as the ipsilateral uptake due to inflammatory changes associated with vocal cord injections. Vocal cord injection is a valuable procedure for the treatment of VCP. Materials for augmentation

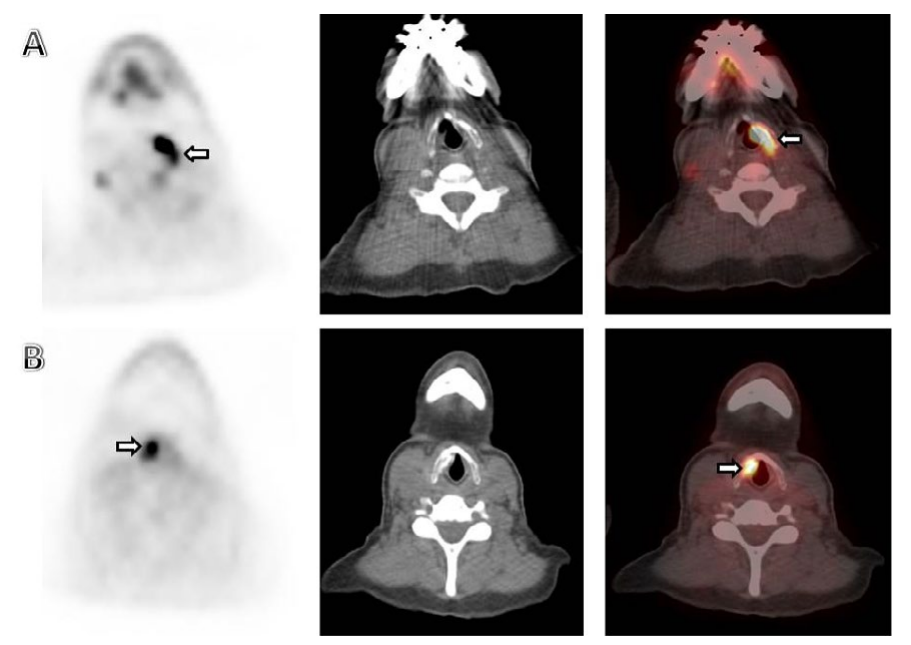

Figure 1. (A) The PET scan showing increased uptake in the left vocal cord (SUV max 3.9), consistent with right vocal cord paralysis.

(B) Post-treatment axial PET, CT and fused PET/CT images showing focal intense FDG uptake in the right vocal cord at the CAHA injection site(SUV 9.2).

Correspondence to: Volkan Beylergil, The Metrohealth Campus of Case Western Reserve University, Department of Radiology, 2500 Metrohealth Drive Cleveland, OH 44108; E-mail: vbeylergil@metrohealth.org

Received: March 10, 2016; Accepted: April 04, 2016; Published: April 08, 2016 


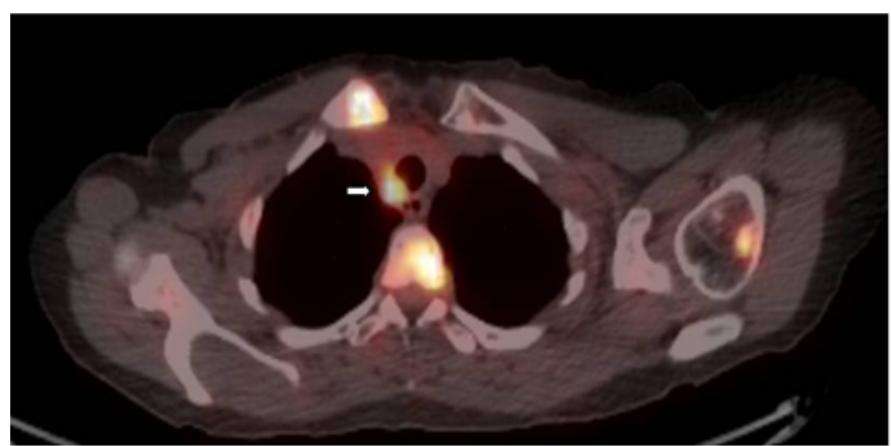

Figure 2. Baseline axial fused PET/CT imaging showing focal intense uptake in the right paratracheal adenopathy, measuring $1.7 \times 1.3 \mathrm{~cm}$ with encroachment of the trachea (SUV 7.9).

are usually classified as either temporary or permanent/long lasting. Long lasting or permanent materials include autologous fat, CAHA, particulate silicone and Teflon. Examples for temporary materials include bovine gelatin, collagen based products, hyaluronic acid and carboxymethylcellulose [6]. Some of the materials can cause substantial immunologic reaction at the injection material [7]. Several case reports have been published, illustrating increased FDG uptake associated with Teflon injection $[4,8]$. Increased FDG uptake in the injected vocal cord is probably due to a granulomatous reaction and may mimic laryngeal cancer [9]. CAHA used as a volumizing filler is known commercially as Radiesse (Bioform Inc, San Mateo, Calif), although canine models have failed to show foreign body reaction to CAHA, Tanna et al. and others have reported serious foreign body reaction which required partial removal of the CAHA material [10]. A few case reports have described focal intense FDG uptake in the paralyzed vocal cord due to previous injection of CAHA [11-13].

Our case is unique in that it demonstrates two important pitfalls in the same patient and supports the fact that CAHA injection may cause paradoxical focal intense FDG uptake in the paralyzed cord.

\section{References}

1. Chen HC, Jen YM, Wang CH, Lee JC, Lin YS (2007) Etiology of vocal cord paralysis.
ORL J Otorhinolaryngol Relat Spec 69: 167-171. [Crossref]

2. Heller MT, Meltzer CC, Fukui MB, et al. (2011) Superphysiologic FDG Uptake in the Non-Paralyzed Vocal Cord: Resolution of a False-Positive PET Result with Combined PET-CT Imaging. Clinical Positron Imaging 3: 207-211.

3. Truong MT, Erasmus JJ, Macapinlac HA, Podoloff DA (2004) Teflon injection for vocal cord paralysis: false-positive finding on FDG PET-CT in a patient with non-small cell lung cancer. AJR Am J Roentgenol 182: 1587-1589. [Crossref]

4. Yeretsian RA, Blodgett TM, Branstetter BF 4th, Roberts MM, Meltzer CC (2003) Teflon-induced granuloma: a false-positive finding with PET resolved with combined PET and CT. AJNR Am J Neuroradiol 24: 1164-1166. [Crossref]

5. Blodgett TM, Fukui MB, Snyderman CH, Branstetter BF 4th, McCook BM, et al. (2005) Combined PET-CT in the head and neck: part 1. Physiologic, altered physiologic, and artifactual FDG uptake. Radiographics 25: 897-912. [Crossref]

6. Mallur PS, Rosen CA (2010) Vocal fold injection: review of indications, techniques, and materials for augmentation. Clin Exp Otorhinolaryngol 3: 177-182. [Crossref]

7. Rosen CA, Gartner-Schmidt J, Casiano R, Anderson TD, Johnson F, et al. (2009) Vocal fold augmentation with calcium hydroxylapatite: twelve-month report. Laryngoscope 119: 1033-1041. [Crossref]

8. Modi D, Fulham MJ, Mohamed A, Havas TE (2005) Markedly increased FDG uptake in a vocal cord after medialization with Teflon: PET/CT findings. Clin Nucl Med 30: 45-47. [Crossref]

9. Ondik MP, Kang J, Bayerl MG, Bruno M, Goldenberg D (2009) Teflon laryngeal granuloma presenting as laryngeal cancer on combined positron emission tomography and computed tomography scanning. J Laryngol Otol 123: 575-578. [Crossref]

10. Tanna N, Zalkind D, Glade RS, Bielamowicz SA (2006) Foreign body reaction to calcium hydroxylapatite vocal fold augmentation. Arch Otolaryngol Head Neck Surg 132: 1379-1382. [Crossref]

11. Damrose EJ (2008) Radiographic properties of injected calcium hydroxylapatite: potential false positive findings on positron emission tomography. J Laryngol Otol 122 1394-1396. [Crossref]

12. Grant N, Sulica L, DeCorato D (2008) Calcium hydroxylapatite vocal fold injectable enhances on positron emission tomography. Otolaryngology--head and neck surgery: Official Journal of American Academy of Otolaryngology-Head and Neck Surgery 138: 807-808.

13. Halpern BS, Britz-Cunningham SH, Kim CK (2011) Intense focal F-18 FDG uptake in vocal cord associated with injection of calcium hydroxylapatite microspheres. Clin Nucl Med 36: e175-177. [Crossref]

Copyright: $\mathbb{C} 2016$ Beylergil V. This is an open-access article distributed under the terms of the Creative Commons Attribution License, which permits unrestricted use, distribution, and reproduction in any medium, provided the original author and source are credited. 